\title{
The fading problem and the population of the Oort cloud
}

\author{
L. Neslušan
}

\author{
Astronomical Institute, Slovak Academy of Sciences, 05960 Tatranská Lomnica, Slovakia \\ e-mail: ne@ta3.sk
}

Received 14 March 2006 / Accepted 29 August 2006

\begin{abstract}
Context. The discovery efficiency of the dynamically new comets has been assumed to be approximately the same as that of all long-period (LP) comets when estimating the population of the Oort cloud. On the other hand, studies of the so-called fading problem have implied a strong difference in the discovery efficiencies of both new and old comets. Some authors have attempted to explain this discrepancy by suggesting that old comets disappeared due to the extinction or disintegration of their nuclei.

Aims. We attempt to answer the question of whether the absolute brightness of old comets steeply decreases in time or whether their nuclei become extinct, dormant, or disintegrated. Moreover, we analyse the impact of the highly different discovery efficiencies on an estimate of the Oort-cloud population.

Methods. The dominance of the fading over the extinction, dormant-phase, or disintegration is demonstrated with the help of the distributions of the reciprocal semi-major axes of the original orbits of the LP comets and a more moderate decrease in discoveries of new comets with increasing perihelion distance. The comet discoveries within the LINEAR sky survey are also used to support our conclusion.

Results. The demonstrated dominance of the fading implies the relatively higher discovery efficiency of new comets. Its ignorance causes an overestimation of the actual intrinsic flux of new comets through the zone of visibility compared to the corresponding intrinsic flux of all LP comets. This overestimate is also documented by a significantly smaller amount of new comets with perihelia $q \geqq 3$ AU discovered within the LINEAR.

Conclusions. The actual intrinsic flux of new comets must be about one order of magnitude lower than has been derived before. Its reduction implies a one-order-of-magnitude less numerous population for the Oort cloud. Moreover, such the reduction also solves (or, at least, weakens) the problem of too high a mass of the comet cloud, as well as the problem of too numerous a population of predicted Halley-type objects or too high a space density of interstellar comets.
\end{abstract}

Key words. comets: general - Oort Cloud

\section{Introduction}

With the discovery of a distant comet cloud, Oort (1950) attempted to explain the distribution of the reciprocal semi-major axis, 1/a, of the original orbits of long-period (LP, hereafter) comets. He noticed that about five times too many comets were presented in the peak of the distribution, corresponding to comets in the cloud (new comets), than was expected from the number of comets having moderate semi-major axes (old comets). He argued that the comets in the peak, being typically on their first passage close to the Sun, may have a greater capacity for developing gaseous envelopes. When such a comet subsequently returns after its supply of volatiles has been depleted, it is considerably fainter and hence may escape detection. Because of this assumption of the fading of comet brightness, the disagreement between the theoretically predicted and observed behaviours of the $1 / a$ distribution became known as the "fading problem". The excess of the predicted old comets was confirmed later by several other authors (e.g. Everhart 1979; Weissman 1979; Wiegert \& Tremaine 1999; Levison et al. 2002; Neslušan 2006).

The related problem that many more Halley-type comets (HTCs) should have been captured from the observed nearparabolic flux than are actually observed has meanwhile been introduced by several groups of authors (Bailey \& Hahn 1992; Hahn \& Bailey 1992; Steel \& Asher 1992; Emel'yanenko \& Bailey 1998; Levison et al. 2001; Napier et al. 2004). More specifically, Emel'yanenko \& Bailey show that the number of Halley-type objects arising from the nearly parabolic cometary flux with absolute magnitudes brighter than $H_{10}=7$ and perihelion distance $q<4$ AU is hundreds of times larger than the number of known HTCs. They were forced to conclude that the observed Halley-type sample is extremely incomplete or that real comets decay rapidly into unobservable remnants, whether as inert cometary asteroids or thin streams of meteoric dust. They did not, however, consider the latter to be a plausible end-state, since comets with $H_{10}<7$ probably have diameters in the range $5-10 \mathrm{~km}$, and such bodies are unlikely to be totally destroyed by outgassing even within a few thousand orbital revolutions. Levison et al. (2002) analysed the LINEAR survey (Stokes et al. 2000) and concluded that the fading problem must be, on the contrary, due to the physical evolution of cometary activity and that missing old comets much more frequently disintegrated entirely rather than becoming extinct or dormant.

In this paper, we offer some evidence that the controversy between the predicted and observed $1 / a$ distributions is caused by the much higher discovery efficiency of the new comets in comparison with the general discovery efficiency of all LP comets. Specifically, in Sect. 2, we demonstrate that the controversy is less when the observed distribution is constructed on the basis of the sample of LP comets with the perihelion distance within $1 \mathrm{AU}$, which has been biased less by the observational selection. Further, we show that the distribution of the perihelion distance of new comets is flatter than that of all LP comets. 
In Sect. 3, we attempt to derive the discovery probability of LP comets within the LINEAR sky survey (Stokes et al. 2000) and point to a serious difficulty calculating this probability due to several uncertain input parameters. Nevertheless, the discoveries of LP comets with $q \lesssim 3$ AU within the LINEAR indicate that the amount of the discovered new comets is, in this case, considerably lower than what is estimated on the basis of a cometary catalogue.

The estimates of the population of the Oort cloud were based on the flux of new comets in the zone of visibility ( $\mathrm{ZV}$, hereafter) corrected with respect to an affection by observational selection effects. In Sect. 4, we present a brief review of several related papers and point out that all authors assumed the same discovery efficiency for both new and old comets. Therefore, the question of what the actual flux of new comets is through the ZV and, consequently, what the actual population of the Oort cloud is turns out not to have been answered properly.

The impact of the different discovery efficiency of new and old comets on an estimate of the Oort-cloud population is analysed in Sect. 5. A summary and discussion of an impact of this different efficiency on some other problems are given in Sect. 6.

\section{Two distributions for the Earth region and entire ZV}

One difference between the discovery efficiencies of both new and old comets, if one exists, is obviously caused by the observational selection that prefers the discovery of one of these two groups of LP comets. This difference could possibly be demonstrated in some situations where the selection effects act in a different way. To prove or disprove the assumption of the same discovery probability, we inspect two orbital characteristics of LP comets, along with their $1 / a$ and $q$ distributions, in this section.

\subsection{The distribution of the reciprocal semi-major axis}

At first, let us construct the $1 / a$ distribution of LP comets with $q<1 \mathrm{AU}$ and compare it with the $1 / a$ distribution of all LP comets. For constructing both distributions, we use the set of original (i.e., before the entrance into the planetary perturbative region) comet orbits recorded in the Catalogue of Cometary Orbits 2003 (Marsden \& Williams 2003). The perihelion distance in the set typically spans up to $\approx 5 \mathrm{AU}$, and the set does not include the sungrazing comets. Both these $1 / a$ distributions are illustrated in Fig. 1. They are gauged so that the highest peak, $0<1 / a<0.0005 \mathrm{AU}^{-1}$, is unity. We can see that the relative number of old comets in the subset of those with $q<1$ AU clearly exceeds the relative number of all old comets. For the sake of transparency, the width of bars in Fig. 1 is chosen to be $0.0005 \mathrm{AU}^{-1}$. If we choose the width of $0.0001 \mathrm{AU}^{-1}$, the highest peak of the distribution can be identified as the Oort peak. Gauging this peak to be, again, unity in both distributions, we can compute the relative number of old comets more exactly. Specifically, the relative number of old comets $\left(0.0001 \mathrm{AU}^{-1}<1 / a<0.029 \mathrm{AU}^{-1}\right)$ in the subset of LP comets with $q<1 \mathrm{AU}$ is 4.42 , while that of old comets in the set of LP comets with every recorded $q$ is 2.16 , i.e. less than one half. This means that if we use a recorded sample that is less affected by the observational selection, the relative number of observed old comets is higher, and the disagreement with the relatively large predicted number of old comets is reduced. The significance of determining the relative numbers can be

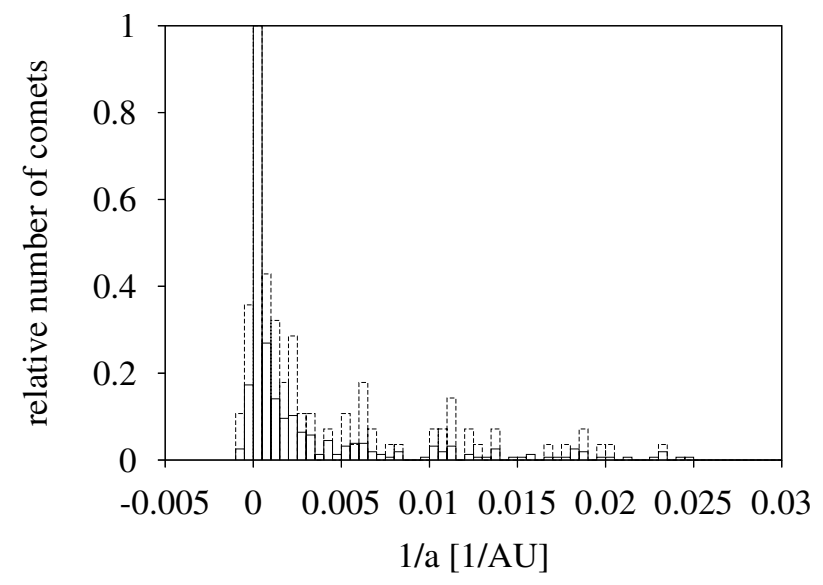

Fig. 1. The relative distribution of the reciprocal semi-major axis, $1 / a$, of known original orbits of LP comets (Marsden \& Williams 2003). The solid-line bars illustrate the distribution for all known LP comets, while the dashed-line bars illustrate it for the subgroup of LP comets with the perihelion distance $q<1 \mathrm{AU}$. Both distributions are gauged so that the relative number of comets in the highest peak, $0<1 / a<0.0005 \mathrm{AU}^{-1}$, becomes unity.

evaluated using the Kolmogorov-Smirnov test (e.g.: http:// www. library. cornell . edu/nr/bookfpdf/f14-3.pdf) for the cumulative counterparts of the distributions in Fig. 1. The test yields a probability of only $10^{-82}$ that both data samples reflect the same distribution, therefore the difference in the relative numbers 4.42 and 2.16 cannot be due to a statistical fluctuation.

In the comparison of the $1 / a$ distributions of two sets of LP comets, we assumed the same distribution of perihelion distance for new and old comets. In reality, we can expect that the physical effects leading to a dormant phase, extinction, or disintegration of old comets depend on the erosive action of solar radiation and solar wind, which increases with decreasing cometary perihelion distance. Statistically, more old comets with smaller $q$ are obviously removed from the orbital-element space of old comets during repeated passages through the ZV. The ordinary old comets with the smallest $q$ are, thus, depleted relative to the new comets with the smallest $q$, which are not significantly influenced by these physical effects before their first perihelion passage. A more specific justification for the dependence of the disruption law on $q$ can be found in the work by Levison et al. (2002). The ratio of old and new comets is, consequently, lower for the comets with small, near-Earth-region perihelia than for the comets with more distant perihelia. In Fig. 1, we would, therefore, observe the opposite situation, i.e. a less relative number of old comets in the subset of LP comets with $q<1 \mathrm{AU}$, if the discovery efficiencies of both new and old comets were equal. Thus, the comparison of the distributions in Fig. 1 reduces the disagreement even more between the predicted and observed numbers of old comets than was evaluated above.

\subsection{The perihelion-distance distribution}

Second, we compare the distributions of the perihelion distance for all LP comets and new comets. We know that the old comets evolve from new comets, whereby the main factors in orbital change are planetary perturbations. Duncan et al. (1987) found that these perturbations change cometary semi-major axes considerably, but they do not change cometary perihelia effectively. According to our recent result (Neslušan 2006), the main perturbing planets, Jupiter and Saturn, typically change the 


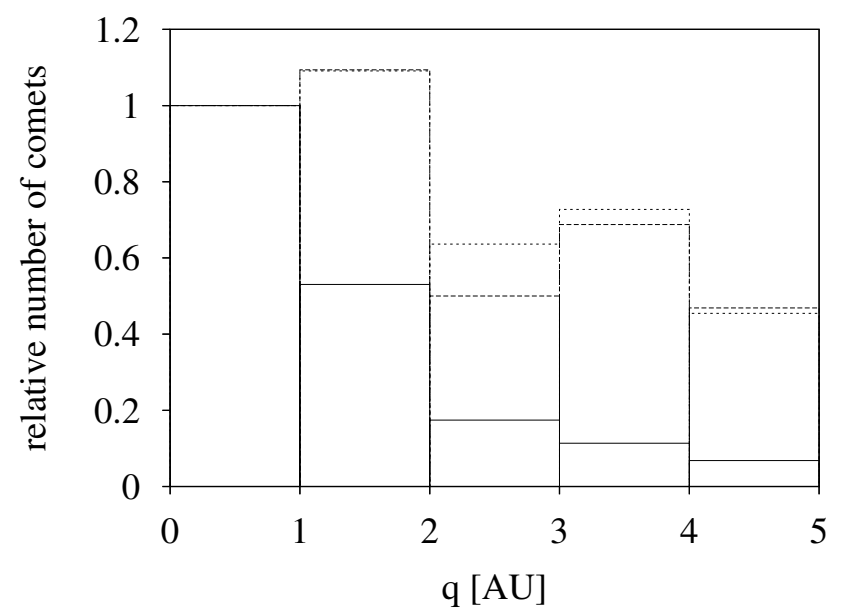

Fig. 2. The relative distribution of perihelion distance, $q$, of all LP comets recorded in the Catalogue of Cometary Orbits (Marsden \& Williams 2003) (solid-line bars) and that of dynamically new comets selected by the $1 / a<10^{-4} \mathrm{AU}^{-1}$ criterion (dashed-line bars) and by Dybczyński's criterion (dotted-line bars). The sungrazing comets and those in a very similar orbit with the orbit of another comet are excluded from the catalogue sample (Neslušan \& Jakubík 2004, Sect. 2.2). All the distributions are gauged to set the relative number of comets in the bar corresponding to $0<q<1$ AU to unity.

perihelia no more than up to about $0.15 \mathrm{AU}$, where the distribution of the change is Gaussian, with the same numbers of both enlarged and reduced perihelia. Therefore, the distribution of $q$ for old comets can be expected to be a conserved $q$ distribution of new comets. A significant difference in the observed $q$-distributions of both cometary subgroups can occur due to the selection effects and, consequently, an unequal discovery probability. The selection effects become more efficient with increasing heliocentric distance, $r$, beyond the Earth's orbit. If the intrinsic brightness of old comets actually fades, then their rate relative to new comets decreases with increasing $r$.

On the other hand, a difference in these $q$-distributions can occur due to the aging of cometary nuclei, which leads to a dormant phase, extinction, or disintegration of old comets. This can reduce their number with the decreasing $q$ as mentioned in Sect. 2.1. A lower intrinsic number of old comets with small $q$ would imply the underestimation of the relative number of all LP comets in Fig. 2, therefore there would be a steeper decrease in their number with increasing $q$ after the appropriate correction and gauging.

Historically, the new comets were distinguished from the old comets by their reciprocal semi-major axis. For new comets, $1 / a<10^{-4} \mathrm{AU}^{-1}$. In 2001, Dybczyński showed, however, that this criterion is insufficient because 41 of 85 comets that were classified by the old criterion as new were likely to be significantly perturbed by the planets at their previous perihelion passage. He regarded only those LP comets as new that had a perihelion distance in their previous perihelion passage that was larger than about $15 \mathrm{AU}$ and that had current $q$ in the ZV. It appears that all such comets have $1 / a<10^{-4} \mathrm{AU}^{-1}$ at the same time. Since the $1 / a<10^{-4} \mathrm{AU}^{-1}$ criterion has been used in many previous works, we still include it in the various comparisons below.

The $q$ distributions of all concerning groups of LP comets are constructed using the Catalogue of Cometary Orbits 2003 (Marsden \& Williams 2003), from which the sungrazing comets and those in a very similar orbit with the orbit of another comet are excluded. A more detailed description of this "homogenization" of the LP-comet sample can be found in work by Neslušan \& Jakubík (2004, Sect. 2.2); the new comets by the Dybczyński's criterion were selected in Neslušan \& Jakubík (2005). The catalogue contains 53 such comets. The distributions are illustrated in Fig. 2. They are gauged to set the number of comets having $q$ in the interval $0<q<1$ AU to unity. The decrease in the relative number of all LP comets for $q>1 \mathrm{AU}$ is clearly steeper than a moderate decrease in new comets, even though the latter are selected either by the $1 / a<10^{-4} \mathrm{AU}^{-1}$ or by Dybczyński's criterion. The relative numbers of new and old comets with $1<q<5 \mathrm{AU}$ are 3.1 and 3.3 for new comets selected by the first and second criteria, respectively, if no erosion of old cometary nuclei is assumed. Since the erosion had to have happened, the actual figures are higher, implying dominance of the selection effects over the processes leading to a disappearance of cometary phenomenon at the nuclei of LP comets.

Converting all three differential distributions to their cumulative counterparts and applying the Kolmogorov-Smirnov test, we can find with a probability of $10^{-11}$ or $10^{-13}$ that the distribution of new comets selected by the first or second criterion, respectively, and distribution of all LP comets are derived from the same intrinsic distribution and that the mutual difference is only an effect of statistical fluctuations in the input data. Such clearly different relative distributions also document an unequal attraction by the selection effects and, consequently, a different discovery efficiency.

\section{Comet discoveries within the LINEAR}

More evidence supporting the conclusion drawn in Sect. 2 can be obtained from the LP-comet discoveries accomplished with the Lincoln Near-Earth Asteroid Research (LINEAR) survey (Stokes et al. 2000) designed to discover unknown near-Earth asteroids, but a few tens of comets have been discovered, too. The discovery probability of near-Earth asteroids within this sky survey was derived by Jedicke et al. (2003). Unfortunately, an analogous determination for LP comets cannot be realized practically because of a large uncertainty on the necessary input parameters. The theoretical steps to the determination are described in more detail in Sect. 3.1, and the uncertainty is discussed in Sect. 3.2. The qualitative dependence of the result on the uncertain parameters is briefly demonstrated in Sect. 3.3. In Sect. 3.4, we use the discoveries of LP comets within the LINEAR to confirm the catalogue's trend in the new-to-old comet ratio and to point out the relative high brightness and, thus, relatively high discovery efficiency of new comets with large heliocentric distances.

\subsection{The calculation of the discovery probability}

In the following, we sketch determination of the partial probability to discover those LP comets with perihelion distance $q$ and absolute brightness $H$, which are situated at heliocentric distance $r$, in one LINEAR observation covering a specified area of the sky. Then, assuming specific distribution laws for $q$ and $H$, we derive the resulting discovery probability.

To account for the observational selection effects of the LP-comet discoveries, we consider some conclusions made by Kresák (1975) in his comprehensive study of the selection effects. According to Kresák, the Holetschek effect disappears for comets with $q>2$ AU. (Holetschek (1891) demonstrated that the number distribution of discovered comets is not constant with respect to an angle, which is the difference between the heliocentric longitudes of comet and Earth, both taken when the comet is 
at its perihelion.) Except for the decrease in discovery efficiency with increasing $q$, the only significant bias in the discoveries of comets is caused by the asymmetric geographic distribution of the observers. Since the directional distribution of LP-comet orbits can be approximated by a random distribution, the distribution of individual objects on the celestial sphere can also be regarded as random, and this sort of bias is not important in the limited-declination sky survey either. Any part of the sky can be chosen to estimate the total number of LP comets.

Comets with $q>2 \mathrm{AU}$ are expected to escape their discovery due to too low an apparent brightness, $m$, which is related to the absolute magnitude, $H$, by relation

$m=H+5 \log _{10} \Delta+2.5 v \log _{10} r$

where $\Delta$ and $r$ are geocentric and heliocentric distances, respectively, and $v$ is the so-called photometric index. Stokes et al. (2000) give the description of the LINEAR survey and its results from the beginning of year 1997 to the end of September 1999. The LINEAR observations were performed not only in a part of sky corresponding to opposition, but also covered the areas well away from opposition. To respect this fact, even if roughly, we replace the usually assumed geocentric distance of observed object $\Delta=r-1$ with a mean distance $\Delta=r-2 / \pi$, where $2 / \pi$ represents the mean projection of the heliocentric distance of Earth along the opposition-adjacent half of its orbit (circular approximation) to the heliocentric radius-vector of the body.

By the symbol $\mathrm{d} p_{1}(q, r, H)$, we denote the partial probability of discovering LP comets, with perihelia within the interval from $q$ to $q+\mathrm{d} q$ and absolute magnitude within the interval from $H$ to $H+\mathrm{d} H$, in the heliocentric distance from $r$ to $r+\mathrm{d} r$ within one LINEAR observation covering the angular sky area $S_{1}$. Let us assume that the distribution function, $f_{q}$, of the flux of LP comets through their perihelion as the function of $q$ is known. This function at the same time gives the influx of comets into a given heliocentric sphere of radius $r_{\text {in }}$. Each comet entering the sphere spends time $t_{\text {in }}$ inside this sphere and time $\tau$ between the spheres of radii $r$ and $r+\mathrm{d} r$. Hence, the probability of finding the comet between $r$ and $r+\mathrm{d} r$ in a given observation is proportional to $\tau / t_{\text {in }}$, and there are

$\mathrm{d} N=f_{q} t_{\text {in }} \mathrm{d} q$

LP comets with perihelia from $q$ to $q+\mathrm{d} q$ inside the sphere of radius $r_{\text {in }}$.

In the number $\mathrm{d} N$, we include all potentially observable LP comets up to a certain upper absolute brightness $H_{\text {up }}$ (see below). A fraction of comets, $\mathrm{d} n / \mathrm{d} N$, with an absolute magnitude from $H$ to $H+\mathrm{d} H$ (where $H \leq H_{\text {up }}$ ) can be determined on the basis of the well-known $H$-distribution law, $\mathrm{d} n(H) \propto 10^{\alpha H} \mathrm{~d} H$, as

$\mathrm{d} n / \mathrm{d} N=\alpha \ln (10) 10^{\alpha\left(H-H_{\text {up }}\right)} \mathrm{d} H$,

where $\alpha$ is the index of the distribution.

Since one LINEAR observation (field of view) covers only the fractional area $S_{1}$ of the sky, the probability that the comet is situated just in this area, is $S_{1} /(4 \pi)$.

Taking all the above partial probabilities into account, the probability of discovering LP comets with perihelia within the interval from $q$ to $q+\mathrm{d} q$ and absolute magnitude within the interval from $H$ to $H+\mathrm{d} H$, in the heliocentric distance from $r$ to $r+\mathrm{d} r$ within one LINEAR observation covering the angular sky area $S_{1}$, is $\mathrm{d} p_{1}=\left[S_{1} /(4 \pi)\right](\mathrm{d} n / \mathrm{d} N)\left(\tau / t_{\text {in }}\right) \mathrm{d} N$, or, explicitly,

$$
\begin{array}{rr}
\mathrm{d} p_{1}(q, r, H)=\frac{\alpha \ln (10)}{4 \pi} S_{1} \tau f_{q} 10^{\alpha\left(H-H_{\text {up }}\right)} \mathrm{d} H \mathrm{~d} q \\
\text { if } m \leq m_{\mathrm{lim}} \\
\mathrm{d} p_{1}(q, r, H)=0 \quad \text { if } m>m_{\mathrm{lim}} .
\end{array}
$$

The overall discovery probability, $p$, within the LINEAR programme can be obtained by multiplying $\mathrm{d} p_{1}$ by the number of performed observations, $n_{\mathrm{obs}}$, and summing all partial probabilities through the entire assumed ranges of $q, r$, and $H$. The product of $S_{1} n_{\text {obs }}$ is equal to the total sky area covered by all observations, $S$.

In Fig. 3, we illustrate the discovery probability as the function of heliocentric distance $r$. To construct a particular plot, we consider a system of concentric spherical layers of thickness $\mathrm{d} r=0.1$ AU having the centre identical to the centre of the Sun. A given comet spends time $\tau$ and has average apparent magnitude $m$ in the layer. The Keplerian orbit with semi-major axis $a_{\mathrm{LP}}$ of the comet is assumed. Since it appears that comets can be discovered only at shorter distances than typically $\approx 15 \mathrm{AU}$, the resulting probability is not very sensitive to this quantity. We consider the value of $a_{\mathrm{LP}}=30000 \mathrm{AU}$ representing a typical semi-major axis of the dynamically new Oort-cloud comets. (We also repeated the calculations for $a_{\mathrm{LP}}=34.2 \mathrm{AU}$, representing the inner border of LP-comet semi-major axis. No significant difference was found.)

In the discovery-probability calculation, we further assume a set of discrete values of $q$ ranging from 2 to $34.2 \mathrm{AU}$ with steps of $0.01 \mathrm{AU}$. The inner limit of $2 \mathrm{AU}$ is chosen to eliminate the Holetschek effect and the outer limit of $34.2 \mathrm{AU}$ is constrained by the inner border of semi-major axes of the LP comets. Having a given $q$, we assume a set of comets with the absolute magnitudes in the range of 0 to $H_{\text {up }}$ (discrete values with steps of 0.1 ). Since $q>2 \mathrm{AU}$, no comet of the considered set can approach the Earth nearer than $1 \mathrm{AU}$, therefore $H_{\text {up }}$ must be lower than the limiting magnitude of the LINEAR survey, $m_{\mathrm{lim}}$. The latter is a value between 18.3 and 19.8 (Jedicke et al. 2003). We approximately identify the value of 19.0 , at which the detection efficiency dropped to $50 \%$ in Jedicke et al., to the limiting magnitude (nominal value). Because $H<m \leq m_{\text {lim }}$ for $r \geq 2 \mathrm{AU}$, it would be meaningless to consider $H>m_{\mathrm{lim}}$, so we put the nominal $H_{\text {up }}=19$.

\subsection{The uncertainty of parameters}

To calculate the realistic discovery probability of LP comets for the LINEAR survey, we need to know the photometric index $v$, the distribution function of their perihelion distance $f_{q}$ (giving the perihelion-passage distribution at the same time), and index $\alpha$ of their absolute-magnitude distribution. Unfortunately, all these parameters have still not be determined very well.

Concerning the photometric index, quondam measurements performed by Vsekhsvyatskij (1925) yielded the value of $v=4$ (see also Vsekhsvyatskij 1958, pp. 26, 27), which has become a standard so we adopt it as a nominal value. We must, however, note that the value of $v$ for an individual comet is supposed to range from 2 to 6 (Krishna Swamy 1986, p. 9). In reality, it can be even more uncertain. Its variation with heliocentric distance can occur even for the same body. Svoreň (1986) measured $v$ for $35 \mathrm{LP}$ comets and found that some outbursts of cometary activity may cause an increase in cometary brightness with $r$ and, thus, lead to a value lower than 2 ( 2 is the value of the index for 

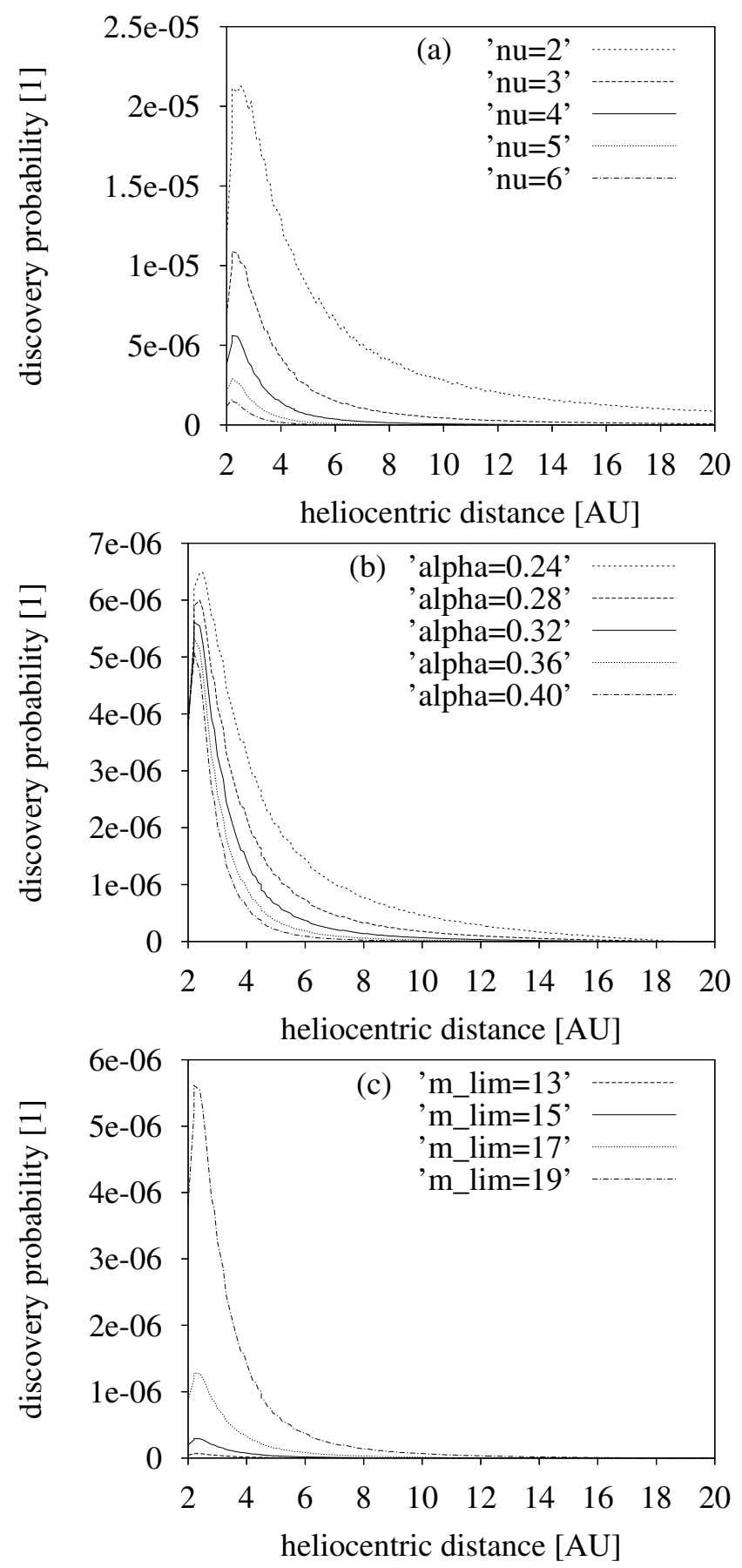

Fig. 3. The discovery probability of the LP comets with $q>2 \mathrm{AU}$ in the intervals of heliocentric distances from $r$ to $r+\mathrm{d} r$, where $\mathrm{d} r=0.1 \mathrm{AU}$, within a single LINEAR-programme observation. In all plots, the solid line illustrates the behaviour of the probability for "nominal" values of uncertain parameters: $v=4, \alpha=0.32$, and $m_{\text {lim }}=15\left(H_{\text {up }}=15\right)$. In plots a) and b), a variation in the probability for changing $v$ and $\alpha$, respectively, is given (the values of the other uncertain parameters are taken to be nominal). In plot c), we compare the behaviours for cometdiscovery sky surveys with the different typical limiting magnitudes, $m_{\lim }$ (again, the other uncertain parameters are nominal). Please notice the different vertical scales of the individual plots. The solid-line curve is the same in all three plots.

an inactive, dark body) in an interval of $r$. On the other hand, such an extreme value as $v=10.9$ was determined. The assumption of constant absolute brightness, which is necessary for acceptance in the process of determining $v$ from the observations, was obviously violated many times.

Svoreň attempted to suppress the brightness fluctuations by dividing the comets into groups by the similarity of the intervals of heliocentric distance, where they had been observed. When the averaged $v$ for a given group was calculated, the contribution of an individual comet of the group entered the calculation with a weight proportional to the length of the observed interval of $r$. Despite this smoothing procedure, one resultant value of the averaged $v$ was still lower than 2 . The interval of the averaged values spanned 1.4 to 4.7 . Surprisingly, no significant difference was found in the indices for the sets of new and old comets. Statistically, the observations more often implied values $v>3$. We must remember note, however, that less than $10 \%$ of all LP comets have been discovered and they are the most active and brightest, so that a typical value of $v$ for all LP comets, not just for the brightest bodies, might be different from any value determined thus far.

The perihelion-distance distribution function, $f_{q}$, was probably suggested first by Everhart (1967), who concluded that the relative number (in arbitrary units) of LP comets with $q$ in the interval from $q$ to $q+\mathrm{d} q$ can be described by (i) the linearly increasing function in the interval from 0 to $\approx 1 \mathrm{AU}$ and a flat behaviour for $q \gtrsim 1 \mathrm{AU}$ and (ii) the linearly increasing function $0.39 q+0.45$ for every $q$. It is likely that the intrinsic distribution is actually somewhere between these two suggested functions. When the intrinsic flux of comets through the perihelion is calculated using this distribution, the constant of proportionality can be derived from Everhart's claim that about 8000 LP comets with $H \leq 10.9$ and $q \leq 4$ AU reached perihelion in 127 years. Therefore, the distribution of the intrinsic flux in the case of function (i) can be given as $f_{q} \mathrm{~d} q=17.1(0.65 q+0.35) \mathrm{d} q$ for $q \leq 1 \mathrm{AU}$ and $f_{q} \mathrm{~d} q=17.1 \mathrm{~d} q$ for $q>1 \mathrm{AU}$. In the case of (ii), $f_{q} \mathrm{~d} q=12.8(0.39 q+0.45) \mathrm{d} q$ for every $q$.

Later, Kresák \& Pittich (1978) suggested that the number is proportional to $q^{1 / 2}$ for $0<q<4$ AU or that the total number of comets, with $q<q_{o}$, passing the perihelion annually, is equal to $2.13 q_{o}^{3 / 2}$ (the so-called equal density model labelled by Kresák $\&$ Pittich with D) implying $f_{q} \mathrm{~d} q=3.195 \sqrt{q} \mathrm{~d} q$. The limiting absolute magnitude of the heterogeneous sample of discovered comets is not unique. The used catalogue was a collection of the comet discoveries, done by the observers equipped with an observational technique with a variable limiting magnitude. It was likely a reason that the authors did not determine any effective limiting absolute magnitude, $m_{\mathrm{lim}}$, but implicitly considered all comets bright enough to be observable in the ZV from the Earth.

Another estimate of the $q$-distribution of the flux was given by Hughes (2001), who suggested its constant behaviour with $q$. The number of LP comets, $n_{H}$, per $1 \mathrm{AU}$ interval of $q$, brighter than $H$ and entering the inner Solar system per year, was expressed as $\log _{10} n_{H}=0.359 H-2.607$.

Utilizing the recent catalogue data, Neslušan \& Jakubík (2004) suggested constraining the LP-comet flux distribution as a function of $q$ between flat and linear behaviours, similar to the Everhart suggestion. Specifically, the flat behaviour can be given as $f_{q} \mathrm{~d} q=3.4 \mathrm{~d} q$ and the linear behaviour as $f_{q} \mathrm{~d} q=5.60 q \mathrm{~d} q$. The constants of proportionality have been derived in more detail, after correcting an observational bias, on the basis of the Catalogue of Cometary Orbits, 15th edition (Marsden \& Williams 2003). Similar to Kresák \& Pittich (1978), all comets recorded in the catalogue were considered, i.e. comets bright enough to be observable from the Earth. A constant 3.4 $\mathrm{AU}^{-1}$ year $^{-1}$ was explicitly specified in the paper (Neslušan \& Jakubík 2005), and 5.60 $\mathrm{AU}^{-2}$ year $^{-1}$ can be obtained, when 
we take the index of slope of the perihelion-passage distribution into account with the bin of $0.2 \mathrm{AU}$ equal to $1.12 \mathrm{AU}^{-2}$ year $^{-1}$ (Neslušan \& Jakubík 2004).

Comparing all suggested $q$-distribution functions, one can demonstrate that Everhart's, Hughes', and Neslušan \& Jakubík's linear distributions give much larger fluxes for LP comets than do the Kresák \& Pittich and Neslušan \& Jakubík flat distributions. It is consistent with the conclusion drawn by Francis (2005) that Everhart's and Hughes' distributions predict many more LP-comet discoveries than were actually the case. The extremely large uncertainty in determining the intrinsic flux is the main reason the discovery probability of LP comets cannot be estimated reliably for the LINEAR.

The index of the LP-comet absolute-magnitude distribution, $\alpha$, has been determined by several authors. For LP comets, Levison et al. (2002) used the same value, $\alpha=0.28 \pm 0.01$, as was found by Weissman \& Lowry (2001) to correspond with the index of the cumulative size-distribution of Jupiter-family and Halley-type cometary nuclei $s=1.40$. Using an alternative method, we derived (Neslušan 2003) a slightly different index $s=1.59$ for exclusively LP comets implying $\alpha=s / 5=0.32$. Though the formal standard deviation of our determination of the index $s$ was only 0.003 , some systematic bias yielded an uncertainty in $s$, and consequently in $\alpha$, of the factor equal to about 1.4. The index $s \doteq 1.60$ corresponding to $\alpha=0.32$ was also found by Hughes (2001), and this value of $\alpha$ is also identical to the $\alpha=0.32 \pm 0.02$ found by Lowry (2001) for the distant Jupiter-family comets. Another value, $\alpha=0.53 \pm 0.05$ for the Jupiter-family comets, was found by Fernández et al. (1999). We can see that no value of $\alpha$ can be regarded as reliable. Moreover, the $H$-distribution law can be broken, as suspected by Francis (2005), where one value of $\alpha$ is valid for $H \lesssim 6$ and another value of $\alpha$ for $H \gtrsim 6$.

\subsection{The behaviour of the discovery probability}

Because of large uncertainty on the input parameters discussed in Sect. 3.2, we only illustrate the behaviour of the probability of the LP-comet discoveries within the LINEAR survey for a few chosen values of $v$ and $\alpha$ and for the flat perihelion-passage-flux distribution suggested by Neslušan \& Jakubík (2004) to demonstrate some qualitative features of this behaviour. The chosen perihelion-passage-flux distribution is roughly consistent with the number of LP-comet discoveries within the LINEAR. To gauge the absolute-magnitude distribution expressed by Eq. (3) in the case of the used perihelion-passage-flux distribution, we adopt a nominal value $m_{\text {lim }} \approx 15$, hence, $H_{\text {up }} \approx 15$. It is a straight average between the value of 10.9 by Everhart and the corresponding limiting LINEAR value of 19 .

The behaviour of the LP-comet-discovery probability is illustrated in Fig. 3. For the values $v=4, \alpha=0.32$, and $m_{\lim }=15$ (these values are chosen as nominal), the behaviour is plotted by the solid line in each plot. In plots (a) and (b), the dependences on changing $v$ and $\alpha$, respectively, are demonstrated. The values of the other quantities are nominal in these plots. For a lower value of $v$ in plot (a), a more moderate decrease in the apparent brightness with $r$ is reflected in a relatively higher discovery probability for higher $r$.

In plot (c), we present the discovery probability as the function of $r$ for several other $H_{\text {up }} \approx m_{\text {lim }}$ than $m_{\text {lim }}=15$. The curve with the highest peak corresponds to the limiting magnitude of the LINEAR, $m_{\text {lim }}=19$. The values of the other quantities are again nominal.

\subsection{The actual ratio of new and old comets}

We can know whether an LP comet is dynamically new or old after determining its original orbit. Any database containing such orbits is, of course, even smaller than the still-not-verynumerous general catalogue of oscullating, often approximate, cometary orbits. Therefore, to estimate the actual number of new comets that passed their perihelion in the $\mathrm{ZV}$ within a certain period, the researchers have usually solved two partial tasks: from the entire sample of all known LP comets, which is statistically better than that of LP comets with known original orbits, they estimated the actual (not only seen) number of all LP comets and then estimated the amount of new comets among all LP comets. It has therefore been necessary to know this amount for any estimate of the actual flux of new comets.

The task when determining the amount of new comets in a sample of all LP comets is, unfortunately, complicated by all the comets that have escaped discovery due to the selection effects during two centuries of collecting for cometary catalogues. The random observations over a long period, which have been conducted by many discoverers by variably efficient techniques, with variably limiting magnitudes, have obviously favoured the discovery of comets with higher absolute brightness.

When the comets were discovered within special sky surveys, with a well-defined limiting magnitude, this effect can be expected to be considerably less, at least for comets with a higher apparent brightness than the limiting magnitude of the given survey. In the following, the evolution of this ratio in time and a comparison of the ratio of new and old comets recorded in the cometary catalogue are shown with an analogous ratio determined on the basis of comets discovered within the LINEAR programme.

Let us denote the number of new (old) comets in a given catalogue by $n_{\text {cat }}\left(l_{\text {cat }}\right)$ and the total number of catalogue LP comets by $s_{\text {cat }}$ (obviously $s_{\text {cat }}=n_{\text {cat }}+l_{\text {cat }}$ ). If the discovery efficiency of new comets is higher than that of old comets and if both efficiencies increase with an improvement in the observational technique in time, it is reasonable to expect a more rapid increase in the lower old-comet discovery efficiency (being even more different from the 100\%) than that of new comets and, consequently, a decrease in ratio $n_{\text {cat }} / l_{\text {cat }}$ with time. Actually, this trend can be documented. When new comets have been distinguished by the $1 / a<10^{-4} \mathrm{AU}^{-1}$ criterion, the ratio found by Oort in 1950 was $n_{\text {cat }} / l_{\text {cat }}=n_{\text {cat }} /\left(s_{\text {cat }}-n_{\text {cat }}\right)=$ $15 /(41-15) \doteq 0.58$. (This ratio was, in reality, even higher, because Oort considered the border between short-period and long-period comets at $1 / a=0.04 \mathrm{AU}^{-1}$ instead of the conventional border of $0.029 \mathrm{AU}^{-1}$.) This can be compared with the $n_{\text {cat }} / l_{\text {cat }}=1 /(3-1)=0.50$ found by Festou et al. in 1993 and with the recent ratio $n_{\text {cat }} / l_{\text {cat }}=111 /(383-111) \doteq 0.41$ counted in the file of original orbits recorded in the catalogue published in 2003 (Marsden \& Williams; see also Neslušan \& Jakubík 2004, Sect. 2.2).

The catalogue ratio $n_{\text {cat }} / l_{\text {cat }}$ corresponds to the comets with relatively short $q$ discovered in the Earth's region. It is documented in Fig. 4, where we show the perihelion-distance distribution of LP comets in the catalogue. This circumstance should be taken into account when we want to compare the catalogue $n_{\text {cat }} / l_{\text {cat }}$ with a corresponding ratio for the LINEAR programme, so let us constrain the "catalogue" ZV by heliocentric distance $\approx 3 \mathrm{AU}$. (This distance seems to be a natural border, not only because of the fall of the corresponding number in Fig. 4, but it is also a critical distance in which a comet approaching the Sun usually starts to exhibit a cometary activity that is due to 


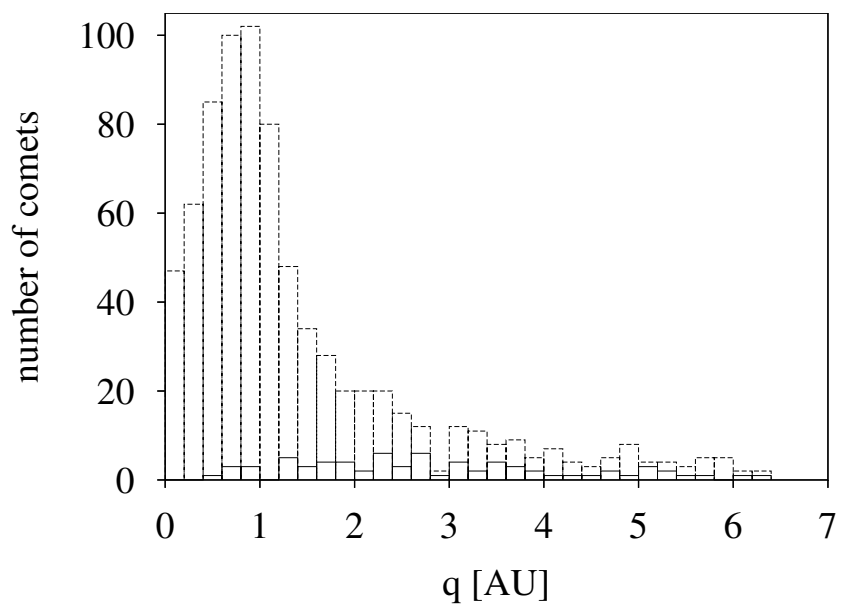

Fig. 4. The distribution of the perihelion distance, $q$, of LP comets constructed on the basis of the Catalogue of Cometary orbits (Marsden \& Williams 2003) (dashed-line bars) and the same distribution constructed on the basis of those LP comets in this catalogue, which were discovered within the LINEAR programme (solid-line bars). The sungrazing comets with $q<0.05 \mathrm{AU}$, as well as other comets in very similar orbits (see Neslušan \& Jakubík 2004 for the details of the homogenization procedure), are omitted.

activation of water vapouring.) Moreover, we consider as "catalogue" only the comets discovered by individual observers and discard those discovered within specialized surveys (LINEAR, NEAT, Spacewatch, LONEOS, BATTERS, Catalina, SWAN) to gain a purer sample of more or less randomly-discovered comets (i.e. with a heterogeneous observational technique).

We can demonstrate that the catalogue ratio $n_{\text {cat }} / l_{\text {cat }}$ differs considerably from the corresponding ratio of both new and old comets, with the perihelia in the catalogue $\mathrm{ZV}$, discovered within the LINEAR. For the catalogue set of data, the ratio $n_{\text {cat }} / l_{\text {cat }}=75 / 185 \doteq 0.41\left(n_{\text {cat }} / l_{\text {cat }}=28 / 232 \doteq 0.12\right)$ for the $1 / a<10^{-4} \mathrm{AU}^{-1}$ (Dybczyński's) criterion. In the LINEAR data set, there are 44 LP comets with the known original orbit, 24 of which have $q<3 \mathrm{AU}$. For this set, the ratio $n_{\text {cat }} / l_{\text {cat }}=$ $4 / 20=0.2\left(n_{\text {cat }} / l_{\text {cat }}=1 / 23 \doteq 0.043\right)$. An enhanced discovery efficiency within the LINEAR leads to a significant decrease in the amount of new comets in the corresponding sample of LP comets, roughly about a factor $\beta \approx 0.41 / 0.2 \approx 2.1$ or $\beta \approx 0.12 / 0.043 \approx 2.8$ for new comets selected by the first or second criteria, respectively.

Unfortunately, the uncertainty in determining this factor is relatively large, therefore we must be careful when drawing a conclusion. Taking the circumstances of the comet discoveries within the LINEAR into account, this uncertainty can be appreciated by applying Poisson statistics. Considering the analogue of the standard deviation $1 \sigma(68.27 \%$ confidence interval $)$ for the Poisson distribution function, we can find the decrease in the factor $\beta$ from $\beta \approx 2.1$ to $\beta \approx 1.2$, when the new comets are selected by the $1 / a<10^{-4} \mathrm{AU}^{-1}$ criterion, and by the decrease from $\beta \approx 2.8$ to $\beta \approx 1.0$ when they are selected by the Dybczyński's criterion. Even at this level of confidence, the factor $\beta$ is close to 1 . In the case of $3 \sigma$ (99.73\% confidence interval), the factor is allowed to decrease well below 1, enabling a clear possibility that the same amount of new comets was discovered as within LINEAR when collecting the catalogue.

The LINEAR survey was probably able to discover almost all new comets with $q<3 \mathrm{AU}$, and its enhanced discovery efficiency was reflected mainly in a higher discovery rate for fainter old comets in this interval of $q$. The enhanced LINEAR efficiency can be seen in Fig. 3c. For example, this efficiency in the distance $r \approx 2.5 \mathrm{AU}$ is about 4 (19) times higher than for a survey with a typical limiting magnitude $m_{\mathrm{lim}}=17$ $\left(m_{\mathrm{lim}}=15\right)$. Concerning the comets with $q>3 \mathrm{AU}$, this survey, however, seems to be efficient at discovering only the brightest comets, which are mostly new comets. This can be proved by the LINEAR analogue to the ratio $n_{\text {cat }} / l_{\text {cat }}$, which is $11 / 9 \doteq 1.22$ or $6 / 14=0.43$ for the first or second criterion, respectively, for LP comets with $q>3 \mathrm{AU}$. Thus, the ratio turns out to be even higher than the recent catalogue ratio for $q<3$ AU. So far, more new comets with this $q$ were discovered than old ones, when the new comets were selected by the $1 / a<10^{-4} \mathrm{AU}^{-1}$ criterion. The idea of brighter absolute magnitudes of new comets in a comparison with the absolute magnitudes of old comets also appears to be supported by LINEAR.

We note that a reflexion of the fading of old comets can be expected in the absolute-magnitude distribution (3), where one value of an index $\alpha$ probably characterises the distribution of new comets $\left(\alpha_{\text {new }}\right)$ and another value of $\alpha$ characterises the distribution of old comets $\left(\alpha_{\text {old }}\right)$. Since the $H$-distribution of new comets is dominated more by the bright bodies, a decrease in the number of bodies with increasing $H$ must be more moderate, i.e. $\alpha_{\text {new }}<\alpha_{\text {old }}$. A qualitative demonstration of a difference in the discovery probability of new and old comets can be seen in Fig. 3b. For example, this probability in the distance $r \approx 4 \mathrm{AU}$ is about 2.3 times higher for the $H$-distribution law with $\alpha=0.28$ than for the law with $\alpha=0.36$.

\section{Oort-cloud-population estimates}

The conclusion about the higher discovery efficiency of new comets compared to old comets and, consequently, all LP comets invalidates the Oort-cloud population estimates based on the assumption of more or less equal efficiency. To reveal the impact of the conclusion on the population estimates done up to the present, we consulted several related papers, but did not find any work that at least tried to distinguish the differences in discovery probability. A brief review of these papers follows.

In the sample of orbital data available to him, Oort (1950) found that there were about 15 new comets in a total of 41 comets with $1 / a<0.04 \mathrm{AU}^{-1}$. Evaluating the completeness of the data in the region of $1 / a<0.04 \mathrm{AU}^{-1}$, these figures implied 83 new comets in the total of 228 for those with $1 / a<0.04 \mathrm{AU}^{-1}$ that had passed through the perihelion between 1850 and 1936, so 97 new comets per century. In the evaluation of the completeness, he took into account all comets with $1 / a<0.04 \mathrm{AU}^{-1}$, without any division into subgroups. Therefore, he did not attempt to distinguish between the completeness of new and old comets and, thus, recognize their different discovery efficiency. Hills (1981) also estimated the population of the outer Oort cloud (Oort outer halo). As far as we noticed, he did however assume an ad hoc rate $N_{c}^{\prime} \approx 5$ per year, at which the comets in completely thermalized orbits in the cloud enter a sphere of radius $q<2 \mathrm{AU}$. Therefore, we cannot discuss the point it makes.

Heisler (1990) derived the actual number of comets with semi-major axes $a>20000 \mathrm{AU}$ from the relative numbers obtained by her numerical modelling, gauging the relative numbers with the observed new-comet flux of 2.1 comets per year for the perihelion distance $q<1 \mathrm{AU}$ and an absolute magnitude brighter than 11. The observed flux was probably calculated using the flux of all LP comets taken from the study by Bailey \& Stagg (1988), which was divided by a factor of 5 giving an average number of orbital revolutions for an originally new comet to be 
removed from the orbital-element space of LP comets. Bailey \& Stagg derived this factor on the basis of the Monte Carlo studies by Weissman (1979). Weissman attempted to match the theoretical $1 / a$ distribution to the observed $1 / a$ distribution and, at the same time, to the theoretical and observed distributions of the LP-comet perihelion distance, simulating the evolution of the LP comets under the influence of a combination of physical (i.e., non-gravitational force, disruptions of cometary nuclei, loss of these nuclei due to planetary collisions, or loss of all volatiles) and dynamical processes. A detailed inspection of his paper can, however, reveal that he had to implicitly assume the same discovery efficiency for both new and old comets and, thus, their equal completeness in the catalogue and in the graph of the observed $1 / a$ distribution. This was, then, reflected in Heisler's estimate of the Oort-cloud population.

To also estimate the outer-Oort-cloud population, Wiegert \& Tremaine (1999) used, in a first step, the fluxes of LP comets found earlier. Specifically, they took the fluxes found by Kresák \& Pittich (1978) and Everhart (1967) into account. Kresák \& Pittich found the rate of 25 LP comets passing within Jupiter's orbit $(5.2 \mathrm{AU})$ per year. Everhart found a value $\approx 60 \mathrm{LP}$ comets with $q \leq 4$ AU per year, which Wiegert \& Tremaine considered, arguing that Everhart took more careful account of selection effects. According to them, about 35 of 60 LP comets would pass their perihelion per year within $3 \mathrm{AU}$. To obtain the flux of new comets, in the second step, the authors utilized the conclusion by Festou et al. (1993) that one in three LP comets is dynamically new. It is clear that it is derived from the rates of new and old comets in the catalogue, without taking the fading effect into account and, consequently, the different discovery efficiency.

Assuming the flat distribution of perihelion distance of LP comets, Neslušan \& Jakubík (2005) determined the completeness of these bodies in the (Marsden \& Williams (2003) catalogue. With this quantity, the flux of all LP comets through the ZV could be determined. When estimating the outer Oort cloud population, these authors simply assumed that the actual ratio of fluxes for new comets and all LP comets through the ZV is the same as the ratio of new comets and all LP comets in the catalogue. The difference between the discovery and recording efficiencies was not noticed.

In a related context, Francis (2005) concluded that the Oort cloud contains many fewer comets than previously estimated. Considering the perihelion-distance and absolute-magnitude distributions of LP comets derived by Everhart (1967) and Hughes (2001), he demonstrated that many more LP comets should have been discovered within the LINEAR survey than actually were. This proves that the expected LP-comet numbers to larger perihelia is not seen and that the number of LP comets per unit of absolute magnitude does not significantly rise to fainter magnitudes. Consequently, the flux of LP comets through the inner solar system is much lower than some previous estimates. Despite this reduction, Francis did not distinguish between the different discovery efficiencies of new and old comets, which would have reduced the flux of new comets and population of the Oort cloud even more.

The Hughes and, especially, Everhart $q$-distributions used by Francis actually predict a considerably higher flux of LP comets through the ZV than the $q$-distribution derived by Kresák \& Pittich (1978) and the flat $q$-distribution derived by Neslušan \& Jakubík (2004). The latter was later used by the authors to estimate the population of the outer Oort cloud, and they (Neslušan \& Jakubík 2005) actually obtained the lowest of all estimates presented here. Specifically, they estimated the population of outer-Oort-cloud comets, which are those bright enough to be observable in the $\mathrm{ZV}$ from the Earth and which semimajor axis $4.35<\log _{10}(a[\mathrm{AU}])<5.05$, to be about $1 \times 10^{11}$ to $2 \times 10^{11}$. Heisler estimated that there are $5 \times 10^{11}$ comets with $a>20000 \mathrm{AU}$ and $H_{10}<11$, and Wiegert \& Tremaine gave the estimate of $1 \times 10^{12}$ comets with $a>20000 \mathrm{AU}$ and $H_{0} \leq 10.9$. Like Heisler but unlike Neslušan \& Jakubík, Wiegert $\&$ Tremaine considered a higher flux of dynamically new comets through the unit (1 AU) interval of perihelion distance in the $\mathrm{ZV}$. Perhaps, it is also worth mentioning that the fluxes by Heisler and Wiegert \& Tremaine were derived for a less extensive ZV (up to 2 and 3 AU, respectively) than Neslušan \& Jakubík considered (up to $6.5 \mathrm{AU}$ ).

\section{Discovery efficiency and Oort-cloud population}

\subsection{The flux corrected with respect to an unequal efficiency}

Let us analyse the impact of the higher completeness rate of new comets in a sample on the estimate of the Oort cloud population. First, we denote the numbers of another three groups of LP comets: $n_{\text {all }}$ denotes all new comets that occurred in the $\mathrm{ZV}$, when a given catalogue was being collected, $l_{\text {all }}$ all old comets passing the $\mathrm{ZV}$ at that time, and $s_{\text {all }}$ all LP comets passing the $\mathrm{ZV}$ then $\left(s_{\mathrm{all}}=n_{\mathrm{all}}+l_{\mathrm{all}}\right)$. We emphasise that the comets that have escaped discovery are also included in the numbers $n_{\text {all }}$, $l_{\text {all }}$, and $s_{\text {all }}$. (When introducing $n_{\text {all }}, l_{\text {all }}$, and $s_{\text {all }}$, we recall that $n_{\text {cat }}\left[l_{\text {cat }}\right]$ is the number of new [old] comets included in the catalogue, and $s_{\text {cat }}$ the number of all LP comets in the catalogue.) We use the term "catalogue" on purpose to distinguish between the real comets with well-determined orbits in a catalogue and the "observed" or "discovered" real comets, some of which can be absent in the catalogue or else their orbits are poorly determined and have to be ignored often in the statistics. The term moreover serves to distinguish between all those real and theoretical (test) comets considered in a simulation.

In the context of the analysis, we follow the procedure by Neslušan \& Jakubík (2005), who expressed the total number of comets in the outer Oort cloud proportional to the number $n_{\text {all }}$. If $\eta=s_{\text {cat }} / s_{\text {all }}$ is the overall completeness of the given catalogue of LP comets and $\eta_{\text {new }}=n_{\text {cat }} / n_{\text {all }}$ is the completeness rate of new comets in the catalogue, whereby the latter is $\rho_{\mathrm{n} / \mathrm{o}}$ times higher than the rate of the completeness of old comets (so, $\eta_{\text {new }}=$ $\rho_{\mathrm{n} / \mathrm{o}} l_{\text {cat }} / l_{\text {all }}$ ), we can, after some handling, easily express $n_{\text {all }}$, with the help of $\eta$ and catalogue numbers, as

$n_{\mathrm{all}}=\frac{1}{\eta} \frac{s_{\mathrm{cat}}}{n_{\mathrm{cat}}+\rho_{\mathrm{n} / \mathrm{o}} l_{\mathrm{cat}}} n_{\mathrm{cat}}$.

For $\rho_{\mathrm{n} / \mathrm{o}}=1$ corresponding to the equal discovery efficiency of new and old comets, number $n_{\text {all }}$ equals $n_{\text {all }}=n_{\text {cat }} / \eta$. It is clear that it must decrease, when $\rho_{\mathrm{n} / \mathrm{o}}>1$ (the discovery efficiency of new comets is higher than that of old comets) and, especially, when $\rho_{\mathrm{n} / \mathrm{o}} \gg 1$.

\subsection{The determination of the discovery-efficiency difference}

The value of quantity $\rho_{\mathrm{n} / \mathrm{o}}$ can be deduced from the disagreement between the number of old comets predicted by constructing the theoretical $1 / a$ distribution and the number of old comets in a catalogue. As already mentioned, Oort (1950) found this ratio equal to $\approx 5$. He assumed the dynamical evolution of the orbits of new comets into the orbits of old comets, accompanied by a cometary-nucleus disruption process. After a certain period, the system reaches a steady state, at which the relative fluxes of new 
and old comets through the $\mathrm{ZV}$ can be predicted. In the steady state, the disruption rate of 0.014 represented the best fit.

Since only the evolution of orbits can be followed reliably, let us separately discuss the upper value of ratio $\rho_{\mathrm{n} / \mathrm{o}}$ (we denote it by $\gamma$ ), which exclusively reflects the dynamical evolution up to reaching the steady state. Value $\gamma \approx 12$ can be deduced from the standard, pure dynamical modelling of the $1 / a$ distribution by Wiegert \& Tremaine (1999). Namely, their simulation produced 35 visible LP comets for each comet in the Oort peak, while the corresponding ratio in the observed sample was 3:1. Those authors used the 8th edition of the Catalogue of Cometary Orbits (Marsden \& Williams 1993).

In our recent work (Neslušan 2006), when aiming to answer the question of whether the fading problem can be solved by assuming a small inhomogenity in the spherical distribution of new-comet orbits, as implied by a new model of the outer Oort cloud (Neslušan \& Jakubík 2005), we modelled the 1/a distribution, considering exclusively the dynamical evolution of the test cometary nuclei, again up to their reaching the steady state. No disruption, fading of brightness, or extinction of nucleus was considered. By this modelling, we found the ratio of predicted and observed old comets (can be identified with $l_{\text {all }} / l_{\text {cat }}$ ) equal to 20 . The number of both predicted and observed new comets was gauged to be unity. Since $\gamma=\left(n_{\text {cat }} / n_{\text {all }}\right) /\left(l_{\text {cat }} / l_{\text {all }}\right)=l_{\text {all }} / l_{\text {cat }}$ with these conditions, the value of 20 can be identified with $\gamma$, so we obtained a higher value than Wiegert \& Tremaine from our modelling and from using the recent, 15th edition of the cometary catalogue (Marsden \& Williams 2003).

It is clear that the lower-than-predicted number of catalogued old comets, in the $1 / a$ distribution, as well as the lower-thanpredicted number of known HTCs, is also a consequence of other effects than their fading brightness. The comets become extinct and, thus, become asteroidal bodies, or else the cometary nuclei disappear due to complete disintegration. Since such comets do not exist, in fact, they cannot be catalogued; therefore, we cannot consider them when we evaluate the completeness of a cometary catalogue. These comets were, however, included in the dynamically modelled $1 / a$ distribution (Neslušan 2006) and had to be the cause of the ratio being $\rho_{\mathrm{n} / \mathrm{o}}<\gamma$.

Nevertheless, the fading of cometary brightness can still be regarded as the dominant cause of the lower discovery and cataloguing efficiency of old comets in comparison with that of new comets. Otherwise, we would see the qualitative opposite situation for the relative numbers of old comets in plots (a) and (b) in Fig. 1, for example. If the fading is dominant, it is reasonable to expect $\rho_{\mathrm{n} / \mathrm{o}}$ to be higher than, at least, $50 \%$ of $\gamma$, from which $10 \lesssim \rho_{\mathrm{n} / \mathrm{o}} \lesssim 20$ (if we consider the value of $\gamma \approx 20$ ) or, simply, $\rho_{\mathrm{n} / \mathrm{o}} \approx 10^{1}$. This is more than the enhancement of the discovery efficiency of old comets, which is about a factor of $\beta \approx 2.1$ to 2.8 within the LINEAR (Sect. 3 ). Even if this uncertain factor was correct, there is no reason to assume that the discovery efficiencies of both new and old comets were perfectly equal even within the LINEAR. Very faint old comets were most probably also not discovered within this survey.

The numbers $n_{\text {cat }}, l_{\text {cat }}, s_{\text {cat }}$, and the discovery-efficiency ratio $\rho_{\mathrm{n} / \mathrm{o}} \approx 10^{1}$ supplied in Eq. (5) imply that the number $n_{\text {all }}$, which is necessary in gauging the theoretical flux of new comets by their observed flux in an Oort-cloud-population estimate, is very likely one order of magnitude lower than derived in the previous works, in which the authors implicitly assumed the value $\rho_{\mathrm{n} / \mathrm{o}}=1$. It leads to a reduction of the Oort cloud population (population of both outer and inner parts and, thus, the entire reservoir, since the population of the inner part is usually estimated as a multiple of the population of the outer part) of about one order of magnitude. The same conclusion is valid for the estimate of the number of HTCs.

\section{Summary and discussion}

The discovery efficiency of new comets recorded in the cometary catalogue has been about one order of magnitude higher than the discovery efficiency of old comets. It can be proved by (i) comparing the $1 / a$ distribution of LP comets with $q<1 \mathrm{AU}$, which are less affected by the observational selection, with the $1 / a$ distribution of all LP comets; for the subset with $q<1 \mathrm{AU}$, there are relatively more old comets; (ii) comparing the $q$-distribution of new comets with that of all LP comets; the relative decrease in new comets with increasing $q$ is more moderate than the relative decrease in all LP comets, in the observed sample; (iii) the fact that a much lower amount of new comets with $q \lesssim 3 \mathrm{AU}$ was discovered within the LINEAR programme with its well-defined limiting magnitude in this region.

The higher discovery efficiency of new comets implies one order of magnitude fewer new comets in the set of all LP comets than believed in past. Consequently, the reduction of the intrinsic flux of new comets through the ZV leads to a one-order-ofmagnitude reduction in the Oort-cloud population.

The two-order-of-magnitude excess, predicted by Emel'yanenko \& Bailey (1998), of actually existing HTCs with respect to the discovered and recorded HTCs can also be explained by the one-order-of-magnitude reduction of the actual flux of new comets. Taking the reduction factor of $\approx 10^{-1}$ into account, the predicted number would not be hundreds of times larger than observed, but only ten or more times larger. Such an excess becomes normal, as the completeness of the cometary catalogue is guessed to be slightly less than $10 \%$ (e.g. Neslušan \& Jakubík 2005). If the excess did not appeared to be larger than two orders of magnitude, no full disintegration of a prevailing part of the missing comets (Levison et al. 2002) would need to be assumed. We would also not be forced to assume that a large fraction of these comets become extinct or dormant. For the sake of completeness, it should also be mentioned that Levison et al. $(2001,2002)$ found the two-order-of-magnitude excess not in the catalogue, but in an already debiased sample of HTCs instead. In this case, the one-order-of-magnitude reduction would not solve, but only weaken, the problem.

Furthermore, we can solve the problem of too high a mass for the Oort cloud, which was mentioned in earlier papers (Neslušan 2000; Neslušan \& Jakubík 2005). This problem is closely related to the problem of the original mass of planetesimals being too high in the solar nebula (Dones et al. 2006). Simulating the formation of the Oort cloud, Dones et al. found the original mass of planetesimals between 4 and $40 \mathrm{AU}$ of some 2 to 8 times the mass of solids in a minimum-mass solar nebula. They considered the population of the outer Oort cloud to be the $5 \times 10^{11}$ published by Heisler (1990) and the average mass of cometary nucleus to be the $4 \times 10^{13} \mathrm{~kg}$ derived by Weissman (1996). Decreasing the Oort cloud population about one order of magnitude, the value of 2 to 8 is also reduced about one order. It thus becomes acceptable.

A higher discovery efficiency of new comets and the consequently lower actual flux of these comets into the $\mathrm{ZV}$ also solves, or at least weakens, the problem of too high a predicted space density for interstellar comets. Namely, Weissman (1990) derived a value that exceeded the upper acceptable limit about 1.3 to 23 times. The one-order-of-magnitude reduction of the Oort cloud population and, in consequence, of the entire small-body population in the once-existing proto-planetary disc is reflected 
in a reduction of the interstellar-comet space density, which thus agrees with the observations better.

Acknowledgements. This work was supported by VEGA - the Slovak Grant Agency for Science, grant No. 7047.

\section{References}

Bailey, M. E., \& Hahn, G. 1992, in Periodic Comets, ed. J. A. Fernández, \& H. Rickman (Montevideo: Universidad de la República), 13

Bailey, M. E., \& Stagg, C. R. 1988, MNRAS, 235, 1

Dones, L., Levison, H., Duncan, M., \& Weissman, P. 2006, Icarus, submitted

Duncan, M., Quinn, T., \& Tremaine, S. 1987, AJ, 94, 1330

Dybczyński, P. A. 2001, A\&A, 375, 643

Emel'yanenko, V. V., \& Bailey, M. E. 1998, MNRAS, 298, 212

Everhart, E. 1967, AJ, 72, 1002

Everhart, E. 1979, in Dynamics of the Solar System, ed. R. L. Duncombe (Dordrecht: Kluwer), 273

Fernández, J. A., Tancredi, G., Rickman, H., \& Licandro, J. 1999, A\&A, 352, 327

Festou, M. C., Rickman, H., \& West, R. M. 1993, Astron. Astrophys. Rev., 5, 37 Francis, P. J. 2005, ApJ, 635, 1348

Hahn, G., \& Bailey, M. E. 1992, in Asteroids, Comets, Meteors 1991, ed. A. W. Harris, \& E. Bowell (Lunar and Planetary Laboratory), 227

Heisler, J. 1990, Icarus, 88, 104

Hills, J. G. 1981, AJ, 86, 1730

Holetschek, J. 1891, Astron. Nachr., 126, 75 (in German)

Hughes, D. W. 1981, MNRAS, 326, 515

Jedicke, R., Morbidelli, A., Spahr, T., Petit, J.-M., \& Bottke Jr., W. F. 2003, Icarus, 161, 17

Kresák, L'. 1975, Bull. Astron. Insts. Czechosl., 26, 92

Kresák, L'., \& Pittich, E. M. 1978, Bull. Astron. Inst. Czechosl., 29, 299
Krishna Swamy, K. S. 1986, Physics of Comets (Singapore: World Scientific Publ. Co.)

Levison, H. F., Dones, L., \& Duncan, M. J. 2001, AJ, 121, 2253

Levison, H. F., Morbidelli, A., Dones, L., et al. 2002, Science, 296, 2212

Lowry, S. C. 2001, Thesis (Belfast: Queen's University)

Marsden, B. G., \& Williams, G. V. 1993, Catalogue of Cometary Orbits, 8th ed. (Cambridge: Smithson. Astrophys. Obs.)

Marsden, B. G., \& Williams, G. V. 2003, Catalogue of Cometary Orbits 2003, 15th ed. (Cambridge: Smithson. Astrophys. Obs.)

Napier, W. M., Wickramasinghe, J. T., \& Wickramasinghe, N. C. 2004, MNRAS, 355,191

Neslušan, L. 2000, A\&A, 361, 369

Neslušan, L. 2003, Contrib. Astron. Obs. Skalnaté Pleso, 33, 5

Neslušan, L. 2006, Contrib. Astron. Obs. Skalnaté Pleso, 36, 5

Neslušan, L., \& Jakubík, M. 2004, Contrib. Astron. Obs. Skalnaté Pleso, 34, 87

Neslušan, L., \& Jakubík, M. 2005, A\&A, 437, 1093

Oort, J. H. 1950, Bull. Astron. Insts. Netherlands, 11, 91

Steel, D., \& Asher, D. J. 1992, in Periodic Comets, ed. J. A. Fernández, \& H. Rickman (Montevideo: Universidad de la República), 65

Stokes, G. H., Evan, J. B., Viggh, H. E. M., Shelly, F. C., \& Pearce, E. C. 2000, Icarus, 148, 21

Svoreň, J. 1986, in Asteroids, Comets, Meteors II, ed. C.-I. Lagerkvist, H. Lundstedt, \& H. Rickman (Uppsala: Reprocentralen HSC), 323

Vsekhsvyatskij, S. K. 1925, AZh (in Russian), 2, 68

Vsekhsvyatskij, S. K. 1958, in Fizicheskie kharakteristiki komet (in Russian) (Moscow: Gossudarstvennoe izdatel'stvo fiziko-matematicheskoj literatury)

Weissman, P. R. 1979, in Dynamics of the Solar System, ed. R. L. Duncombe (Dordrecht: Kluwer), 277

Weissman, P. R. 1990, Nature, 344, 825

Weissman, P. R. 1996, in Completing the Inventory of the Solar System, ASP Conf. Ser., 107, ed. T. W. Retting, \& M. Hahn (Astron. Soc. Pacific), 265

Weissman, P. R., \& Lowry, S.C. 2001, Bull. Am. Astron. Soc. DPS meeting \#33, \#31.04

Wiegert, P., \& Tremaine, S. 1999, Icarus, 137, 84 